\title{
Thoracocervical cysts as a cause of stridor
}

\author{
B. T. le ROUX, B. J. HENDERSON, E. U. KÜRKLÜ, \\ and M. A. ROGERS
}

The Thoracic Unit, Wentworth Hospital, Durban, and the Department of Surgery, University of Natal

\begin{abstract}
le Roux, B. T., Henderson, B. J., Kürklii, E. U., and Rogers, M. A. (1974). Thorax, 29, 564-566. Thoracocervical cysts as a cause of stridor. In five patients with severe stridor four were shown to have as the cause a duplication of foregut and the fifth a cystic hygroma. All were managed surgically and are well. In one patient tracheal obstruction was so severe that asphyxia and an episode of cardiac arrest preceded surgical management. Foregut duplication, particularly in children, is an important and remediable cause of respiratory obstruction.
\end{abstract}

In a seven-year period during which 82 patients with mediastinal cysts and tumours were managed surgically, five presented because of severe stridor. One patient experienced complete respiratory obstruction shortly after admission to hospital, followed by anoxic cardiac arrest. Resuscitation was successful after endotracheal intubation. In four patients the cause of tracheal obstruction was a duplication of foregut; in the fifth the lesion was a thoracocervical cystic hygroma. All were managed surgically and are well.

\section{CASE HISTORIES}

CASE 1 An African female of 20 months had experienced respiratory symptoms including wheeze for four months and had been urgently stridulous for a week before admission to hospital. Clinically a tense, cystic mass was palpable in the suprasternal notch. Radiographically the upper mediastinum was broad and the trachea slit-like and displaced to the right (Fig. 1). Through a transverse cervical incision a cyst was enucleated from the superior mediastinum. The histological features were those of foregut duplication. Stridor was relieved and convalescence uneventful.

CASE 2 An African male child of 3 months was admitted with a story of ingravescent stridor over four weeks. Radiographically there was a well-defined opacity, convex laterally, flattened against the trachea and occupying most of the upper half of the right hemithorax. The trachea was displaced to the left and compressed. At right thoracotomy a tense cyst, the size of a tennis ball and shown to contain $100 \mathrm{ml}$ of thick green mucus, was dissected off the trachea. The histological features were those of foregut duplication with a lining predominantly of ciliated columnar epithelium.

CASE 3 An African child of 11 months had a history, radiological features, and surgical findings indistinguishable from those of case 2 .

CASE 4 An African man of 22 years was transferred from an outlying hospital with a history of increasing stridor. Accompanying radiographs (Fig. 2) showed the trachea to be displaced to the right and the upper mediastinum to be broad. Asphyxia and cardiac arrest shortly after admission were successfully managed. An endotracheal tube was left in situ for 24 hours, by which time it was clear that recovery from the episode of cardiac arrest was complete. The endotracheal tube was removed; stridor returned and was so severe that the endotracheal tube was reinserted. Without a precise diagnosis in mind, it was elected to proceed to diagnostic exploration, probably through a vertical sternotomy. After induction of anaesthesia, when the relaxant became effective and with positive-pressure ventilation, there appeared from the mediastinum what could only be a cyst, which herniated into the neck, giving a frog-like appearance. Through a transverse cervical incision a cyst which $\omega$ was anchored to the third tracheal ring was enucle- $\underset{\gamma}{\sigma}$ ated. Convalescence was uneventful; histologically the lesion was a foregut duplication.

CASE 5 An African woman of 34 years complained of dyspnoea and a swelling in the left supraclavicular fossa. She was stridulous. Radiographically (Fig. 3) there was a large, well-defined, and predominantly left-sided opacity with a clear-cut lateral margin. A biopsy made from the soft fluctuant mass in the left 


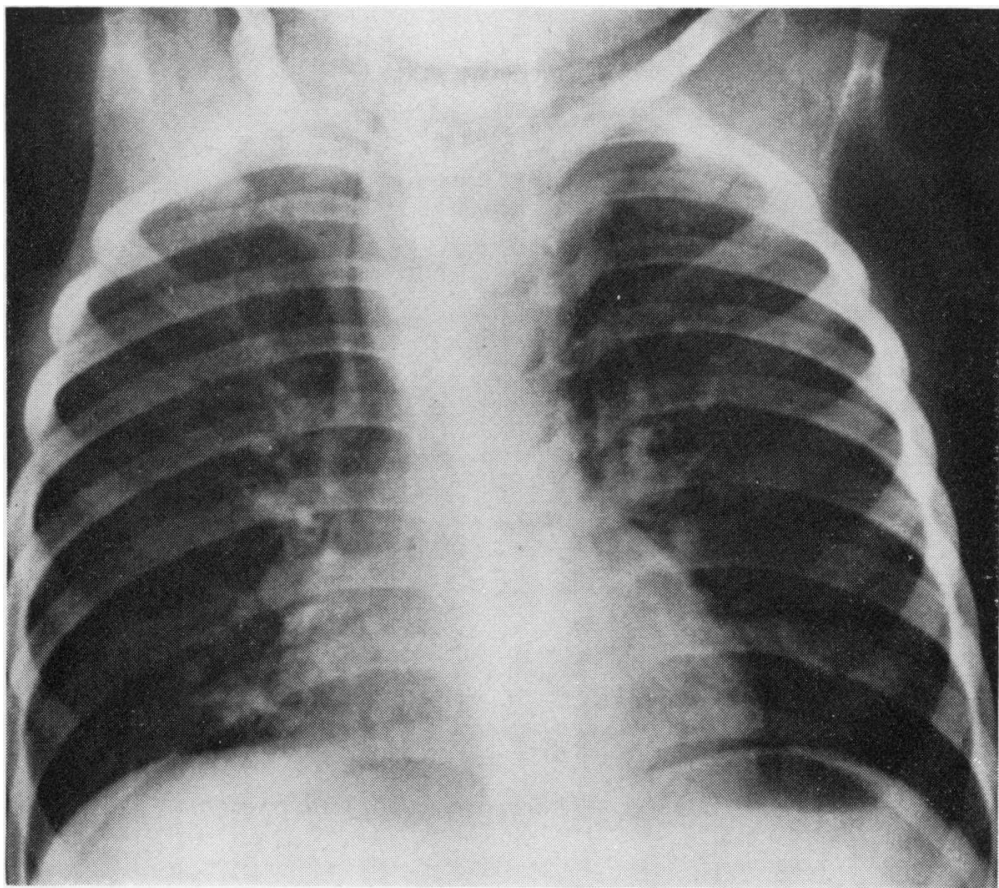

FIG. 1. Case 1. Postero-anterior chest radiograph showing tracheal narrowing and displacement to the right consequent upon a thoracocervical foregut duplication.

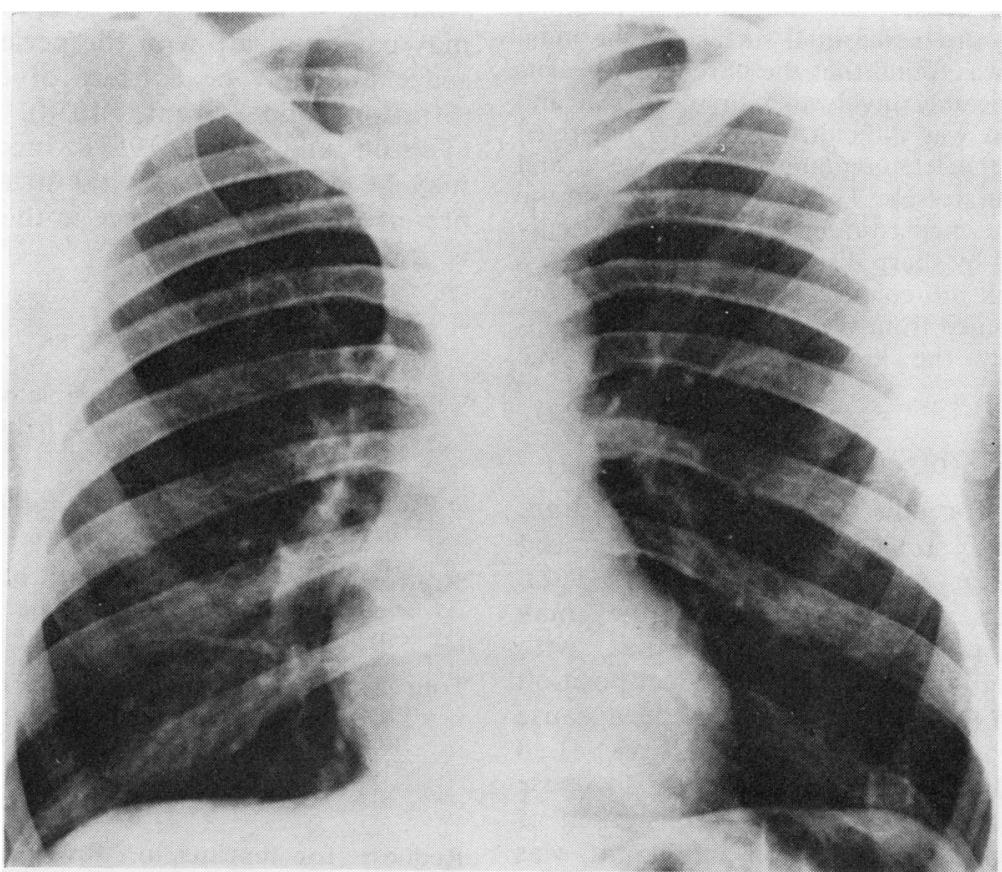

FIG. 2. Case 4. The broad mediastinum above the aortic arch is well seen. 


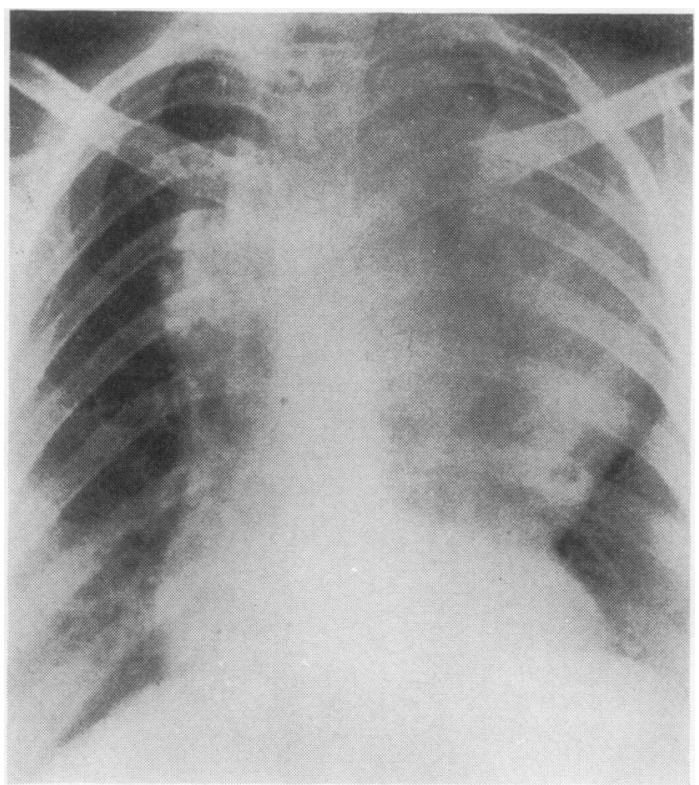

FIG. 3. Case 5. Thoracocervical cystic hygroma.

supraclavicular fossa showed the features of lymphangioma or cystic hygroma.

At left thoracotomy there was demonstrated in the superior mediastinum a large, soft, cystic mass with ill-defined borders, densely adherent to the mediastinal structures and to the mediastinal surface of the lung. On dissection it was found that the phrenic and vagus nerves were intricately involved with the lesion and their mobilization was difficult. The lesion encircled the aortic arch, the left common carotid artery, and the left subclavian vessels. The cyst extended into the right side of the mediastinum whence it was only partially removed by sharp dissection. The cystic mass was spongy, pink in colour, and contained turbid liquid. Convalescence from thoracotomy was uneventful. Histologically the lesion was called a cystic hygroma.

\section{DISCUSSION}

Duplications of foregut, particularly in children, may achieve large size and are a well-recognized cause of respiratory obstruction (le Roux, 1962). It is perhaps less well recognized that they may occur so high in the mediastinum that, with growth, they can adopt a thoracocervical position and may, from tracheal obstruction, be a cause of death. The behaviour of the cyst (case 4) so firmly impacted in the thoracic inlet as to cause asphyxia and cardiac arrest, on establishment of positive-pressure ventilation with a relaxant, was remarkable to observe.
Cystic hygroma in the mediastinum is rare and is usually thoracocervical (Camishion and Tem- $-\mathbb{D}$ pleton, 1962). It may achieve large size and is a well-recognized cause of the 'superior mediastinal' syndrome with respiratory symptoms. An hygroma may communicate with the pericardial or pleural space and may be a cause of chylopericardium (Stratton and Grant, 1958) or chylothorax (Touroff and Seley, 1953). Incomplete excision $\underset{\times}{\stackrel{D}{N}}$ may be a necessity if vital structures are to be preserved. Late recurrence in these circumstances is unusual.

\section{REFERENCES}

Camishion, R. C. and Templeton, J. Y. (1962). Cervicomediastinal cystic hygroma. Paediatrics, 29, 831.

le Roux, B. T. (1962). Intrathoracic duplication of $\mathrm{N}$ the foregut. Thorax, 17, 357.

Stratton, V. C. and Grant, R. N. (1958). Cervicomediastinal cystic hygroma associated with chylopericardium. Archives of Surgery, 77, 887.

Touroff, A. S. W. and Seley, G. P. (1953). Chronic $\stackrel{\text { ? }}{+}$ chylothorax associated with hygroma of the mediastinum. Journal of Thoracic Surgery, 26, 318.

Requests for reprints to: Professor B. T. le Roux, The Thoracic Unit, Wentworth Hospital, Durban. 